\title{
Trapping Balloon Technique for Removal of the Burr in Rotational Atherectomy
}

\author{
Kei Yamamoto, ${ }^{1}$ MD, Kenichi Sakakura, ${ }^{1}$ MD, Yousuke Taniguchi, ${ }^{1}$ MD, Yoshimasa Tsurumaki, ${ }^{1}$ MD, \\ Hiroshi Wada, ${ }^{1}$ MD, Shin-ichi Momomura, ${ }^{1}$ MD and Hideo Fujita, ${ }^{1}$ MD
}

\begin{abstract}
Summary
Because rotational atherectomy (RA) has several unique complications, such as burr entrapment, vessel perforation, and slow flow, it is important for interventional cardiologists to be familiar with bailout procedures for such complications. The principal part of bailout procedures is to keep a guidewire in the target coronary artery during the procedure. However, it is not easy to keep a guidewire in the same position during the removal of a burr because the length of the RA guidewires is $300 \mathrm{~cm}$, and the removal of a burr requires collaboration between the primary operator and an assistant. We describe the case of an 83-year-old male with stable angina. We performed RA to the left anterior descending artery, and removed the burr using a KUSABI (Kaneka Medix Corporation, OSAKA, Japan) trapping balloon technique without activating the dynaglide mode. This simple technique would help RA operators remove a burr more reliably than the conventional removal technique.
\end{abstract}

(Int Heart J 2018; 59: 399-402)

Key words: Percutaneous coronary intervention

$\mathrm{B}$ ecause the prevalence of diabetes mellitus or chronic renal failure on hemodialysis is increasing, ${ }^{1,2}$ the incidence of complex calcified lesions is also increasing in the current percutaneous coronary interventions (PCI). ${ }^{3)}$ Rotational atherectomy (RA) is mostly used for lesion modifications in severely calcified lesions in the current PCI. ${ }^{4,5)}$ Because RA has several unique complications, such as burr entrapment, vessel perforation, and slow flow, it is important for interventional cardiologists to be familiar with bailout procedures for such complications. ${ }^{6-10)}$ The principal part of the bailout procedure is to keep a guidewire in the target coronary artery during the procedure, which enables the operator to bring a perfusion balloon or a covered stent to the lesion when lifethreatening complications occur. ${ }^{6}$

However, it is not easy to keep a guidewire in the same position during the removal of a burr because the length of the RA guidewires (RotaWire Floppy ${ }^{\mathrm{TM}}$ guidewire or RotaWire Extra Support ${ }^{\text {TM }}$ guidewire) is 300 $\mathrm{cm}$, and the removal of a burr requires collaboration between the primary operator and an assistant. Therefore, reliable methods should be sought to help operators remove a burr, while keeping a guidewire. We present a case of a calcified lesion that was treated using RA. A trapping balloon technique was used for removing a burr from the coronary artery, which has not been used in RA.

\section{Case Report}

An 83-year-old male with a history of pacemaker implantation and vasospastic angina was referred to our medical center because of stable angina. As a coronary angiogram showed significant stenosis at the middle segment of the left anterior descending artery (LAD; Figure 1 A), we performed an elective PCI to the LAD. A 7-Fr CLS 3.5SH guide catheter was inserted via the left radial artery. A conventional 0.014-inch guidewire was advanced and intra-vascular ultrasound (IVUS) was performed. Since the IVUS revealed circumferential $\left(>270^{\circ}\right)$ superficial calcification (Figure 1B), we decided to perform RA. After exchanging the conventional guidewire with a RotaWire Floppy ${ }^{\mathrm{TM}}$ guidewire using a microcatheter and a KUSABI trapping balloon catheter (Kaneka Medix Corporation, OSAKA, Japan), the lesion was ablated using a 1.5 $\mathrm{mm}$ burr (Figure 1C). Because the guidewire position was not distal enough at the time of burr removal, loss of the guidewire from the coronary artery was feared. Therefore, the KUSABI trapping balloon catheter was inserted via a Y-connector and the RotaWire Floppy ${ }^{\mathrm{TM}}$ guidewire was fixed after pulling the burr halfway out of the guide catheter. Because the RotaWire Floppy ${ }^{\mathrm{TM}}$ guidewire was fixed well (Figure 1D), we easily pulled out the burr without activating the dynaglide mode. Following RA, we deployed a biodegradable polymer everolimus-eluting stent (Figure 1E) and obtained a favorable result (Figure 1F).

From the ${ }^{1}$ Division of Cardiovascular Medicine, Saitama Medical Center, Jichi Medical University, Saitama, Japan.

This work was supported by Grants-in-Aid for Scientific Research (C) (Kenichi Sakakura, JSPS KAKENHI Grant Number 17K09521).

Address for correspondence: Kenichi Sakakura, MD, Division of Cardiology, Department of Cardiovascular, Saitama Medical Center, Jichi Medical University, 1-847 Amanuma-cho, Omiya-ku, Saitama-city, Saitama 330-8503, Japan. E-mail: ksakakura@jichi.ac.jp

Received for publication June 27, 2017. Revised and accepted August 18, 2017.

Released in advance online on J-STAGE February 20, 2018.

doi: 10.1536/ihj.17-359

All rights reserved by the International Heart Journal Association. 

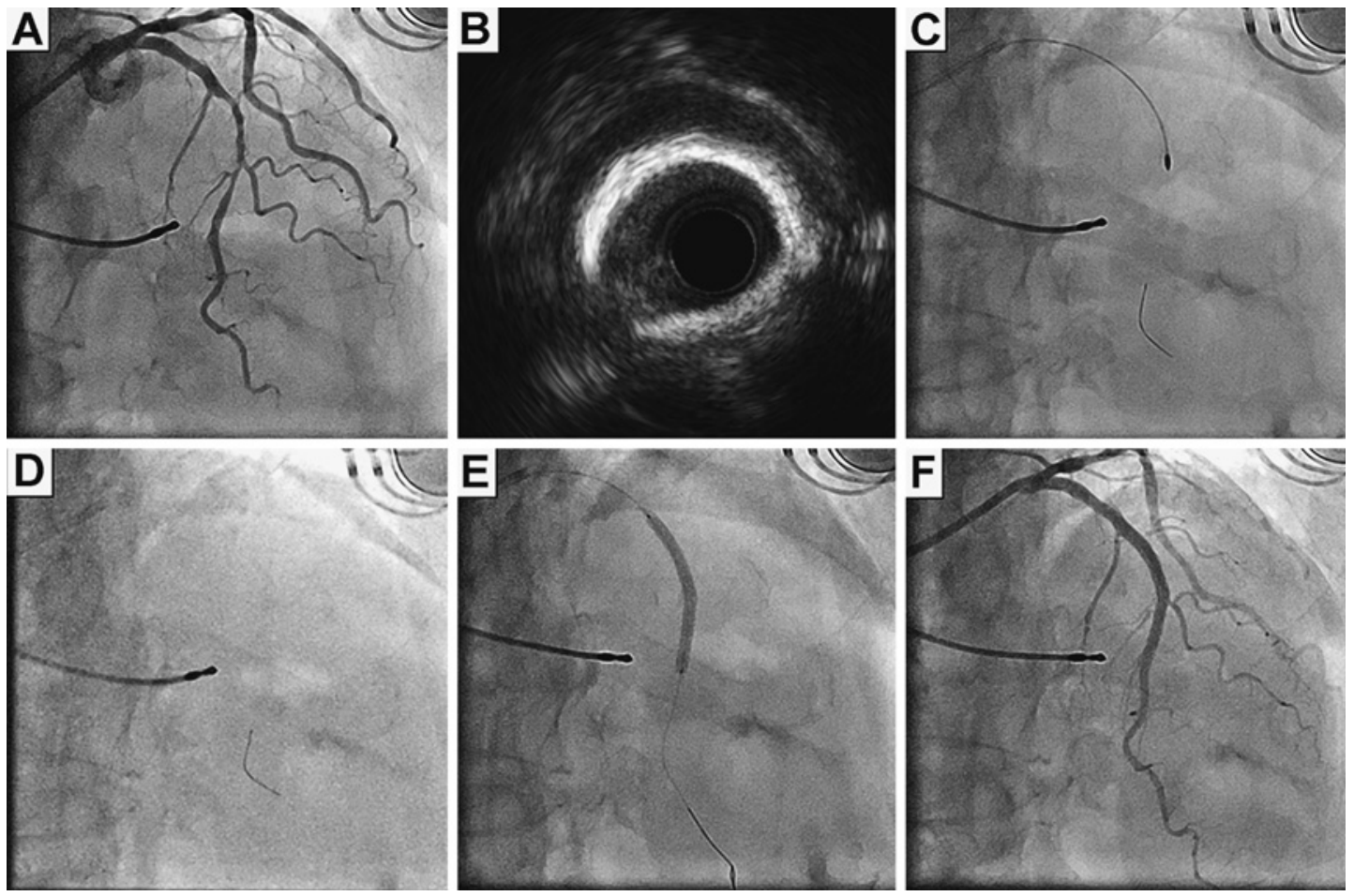

Figure 1. A: Coronary angiography shows calcified stenosis in the middle of the left anterior descending artery. B: Intravascular ultrasound shows circumferential calcification. C: The $1.5 \mathrm{~mm}$ burr passed the calcified lesion. D: RotaWire was fixed using a KUSABI trapping balloon catheter during the removal of the burr. E: Everolimus-eluting stent $(3.0 \times 38 \mathrm{~mm})$ was deployed. F: Final angiogram.

Written informed consent for this case report was obtained from this patient after the procedures.

After the above case, we performed this procedure in 19 additional cases (total 20 cases). We attempted this procedure using a $1.25 \mathrm{~mm}$ burr or $1.5 \mathrm{~mm}$ burr in $\geq 7-\mathrm{Fr}$ guide catheters. The procedure was not attempted using a $1.75 \mathrm{~mm}$ burr or $2.0 \mathrm{~mm}$ burr because a strong resistance was encountered when a $1.75 \mathrm{~mm}$ burr was used in the 7 Fr guide catheter in a bench test. In 20 cases, only one case of unsuccessful procedure was observed in which the guidewire was not fixed securely by the KUSABI trapping balloon catheter. Thereafter, we switched from the KUSABI trapping balloon technique to the conventional removing process. Therefore, a procedure success rate was $95 \%$. Details of the 20 cases are shown in the Table. This retrospective analysis of 20 cases was approved by the institutional review board, and written informed consent for the additional 19 cases was waived because of the retrospective study design.

A bench test was also performed to show the mechanism of this technique. We inserted a $1.5 \mathrm{~mm}$ burr via a RotaWire into a 7-Fr FR3.5SH guide catheter in a dry model (Figure 2A). Subsequently, a KUSABI trapping balloon catheter was inserted beyond the $1.5 \mathrm{~mm}$ burr (Figures 2B, 2C). After the KUSABI trapping balloon catheter overtook the $1.5 \mathrm{~mm}$ burr, it was inflated to 14 atm (Figure 2D). Finally, the $1.5 \mathrm{~mm}$ burr was pulled out without activating the dynaglide mode (Figure $2 \mathrm{E}$ and 2 F). The bench test was repeated six times and that a sin- gle KUSABI trapping balloon catheter was workable in six consecutive attempts was confirmed.

\section{Discussion}

In this case report, we extended the usefulness of the KUSABI trapping balloon catheter for the removal of RA burrs. Although the KUSABI trapping balloon catheter was invented to trap the guidewire during the removal of PCI devices, such as a microcatheter, a double lumen catheter, or an over-the-wire balloon catheter, it has not been used for the removal of RA burrs. There were several reasons why the KUSABI trapping balloon catheter was not used for RA. First, the RA burrs are usually pulled out by activating the dynaglide mode, while the RA burrs must be pulled out without activating the dynaglide mode once the KUSABI trapping balloon catheter has fixed the RotaWire firmly. Second, RA was launched more than 20 years ago, while the KUSABI trapping balloon catheter is a relatively new device launched a few years ago. The manufacturers of each device did not expect that the KUSABI trapping balloon catheter would be workable during RA. Third, the conventional trapping balloon technique by 2.0-3.0 mm balloons was not used during the removal of RA burrs because those balloons usually cannot overtake the RA burrs within a guide catheter. While the trapping balloon technique for general use is workable for conventional $2.0-3.0 \mathrm{~mm}$ balloons as well as the KUSABI trapping balloon catheter, the KUSABI trap- 
Table. Procedure Characteristics of 20 Cases

\begin{tabular}{rccccc} 
Case & Target lesion & $\begin{array}{c}\text { Guide catheter } \\
\text { size }(\mathrm{Fr})\end{array}$ & $\begin{array}{c}\text { Type of } \\
\text { RotaWire }\end{array}$ & $\begin{array}{c}\text { Burr size } \\
(\mathrm{mm})\end{array}$ & $\begin{array}{c}\text { Procedure } \\
\text { success }\end{array}$ \\
\hline 1 & Mid LAD & 7 & Floppy & 1.5 & Success \\
2 & Proximal LAD & 7 & ExtraSupport & 1.25 & Success \\
3 & Proximal RCA & 7 & Floppy & 1.25 & Success \\
4 & Left main & 7 & Floppy & 1.5 & Success \\
5 & Proximal RCA & 8 & Floppy & 1.25 & Success \\
6 & Proximal RCA & 7 & Floppy & 1.25 and 1.5 & Success \\
7 & Proximal RCA & 7 & ExtraSupport & 1.25 & Success \\
8 & Proximal LAD & 7 & ExtraSupport & 1.25 & Success \\
9 & Proximal RCA & 7 & Floppy & 1.5 & Failure \\
10 & Proximal LAD & 7 & Floppy & 1.25 & Success \\
11 & Proximal LAD & 7 & ExtraSupport & 1.5 & Success \\
12 & Mid RCA & 8 & Floppy & 1.5 & Success \\
13 & Left main & 7 & Floppy & 1.5 & Success \\
14 & Proximal LAD & 7 & Floppy & 1.5 & Success \\
15 & Proximal RCA & 7 & Floppy & 1.25 & Success \\
16 & Proximal LAD & 7 & Floppy & 1.25 & Success \\
17 & Proximal LAD & 7 & Floppy & 1.25 & Success \\
18 & Proximal RCA & 7 & Floppy & 1.5 & Success \\
19 & Proximal LCX & 8 & Floppy & 1.25 & Success \\
20 & Left main & 7 & Floppy & 1.5 & Success \\
\hline
\end{tabular}

LAD indicates left anterior descending artery; RCA, right coronary artery; and LCX, left circumflex artery.
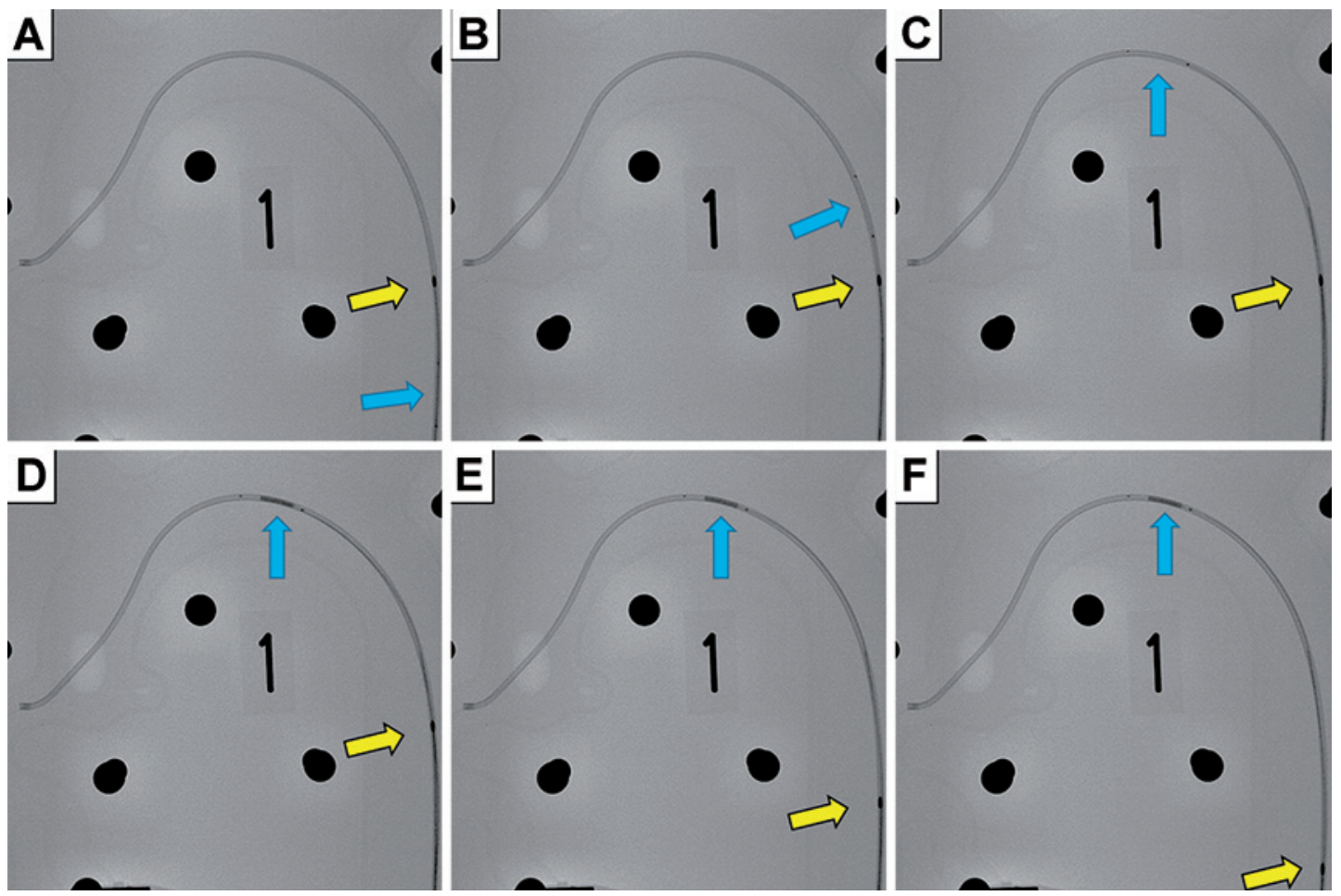

Figure 2. A $1.5 \mathrm{~mm}$ burr via RotaWire into a 7-Fr FR3.5SH guide catheter in a dry model. Yellow arrows show the burr, and blue arrows show the KUSABI trapping balloon catheter. A: KUSABI trapping balloon is positioned distal (Y-connector side) to the burr. B and C: The KUSABI trapping balloon overtook the burr and was positioned proximal (orifice of guide catheter side) to the burr. D: KUSABI trapping balloon was inflated to $14 \mathrm{~atm}$. E and $\mathbf{F}$ : The burr was pulled out without activating the dynaglide mode.

ping balloon catheter has a shaft profile smaller than the conventional balloons because there is no guidewire lumen. Thus, the KUSABI trapping balloon catheter can overtake the RA burrs within a guide catheter because of its small shaft profile.

There are some clinical implications for this tech- 
nique. The most catastrophic complication during RA is vessel perforation, especially Type III perforation. ${ }^{6}$ The incidence of cardiac tamponade and emergent surgeries in RA is reported to be $0.64 \%$ and $0.18 \%$, respectively, which is greater than those in conventional PCI. ${ }^{11)}$ If we could not maintain the guidewire during the removal of the burr in Type III perforation, it would be extremely challenging to conduct the bailout procedures because reinsertion of the guidewire to the perforated (or ruptured) vessel is sometimes impossible. Because RA operators know the importance of keeping the guidewire in severe complications, they would experience considerable pressure during the removal of the burr when vessel perforation is suspicious during RA. Therefore, any technique for keeping the guidewire during the removal of the burr would be helpful for RA operators. Moreover, since the KUSABI trapping balloon catheter is often used in RA for the purpose of guidewire exchange by using a microcatheter, it would not generate an additional cost during the removal of the burr.

This KUSABI trapping balloon technique has several limitations. First, we cannot use this technique for large burrs $(\geq 1.75 \mathrm{~mm})$ in the $7-\mathrm{Fr}$ system. Also, we did not check the availability of this technique in the 6-Fr system. Second, the burr must be pulled out halfway of the guide catheter in the dynaglide mode before the KUSABI trapping balloon catheter is inserted because the KUSABI trapping balloon catheter has to overtake the burr within the guide catheter. Third, friction between the balloon and the diamond coating of the burr may damage the KUSABI trapping balloon catheter, which can cause trapping balloon rupture. Finally, although we do not have any experience that the RotaWire was broken or stretched during this procedure, we should be careful about damages to RotaWires in this KUSABI trapping balloon technique because RotaWires are usually more fragile than conventional guidewires. If RA operators would like to reuse the same RotaWire for additional ablations, including burr size up, they should check any damages to the RotaWire before additional ablation.

In conclusion, the KUSABI trapping balloon technique for the removal of the burr is a new technique in RA. This simple technique would help RA operators to remove the burr more reliably than the conventional removal technique and would enhance the safety of RA.

\section{Disclosures}

Conflicts of interest: Dr. Sakakura has received speaking honoraria from Abbott Vascular, Boston Scientific, Medtronic Cardiovascular, Terumo, OrbusNeich, and NIPRO; has served as a proctor for Rotablator for Boston Scientific; and has served as a consultant for Abbott Vascular and Boston Scientific.

\section{Acknowledgments}

The authors acknowledge all staff in the catheter laboratory in Saitama Medical Center, Jichi Medical University for their technical support in this study.

\section{References}

1. Sterling SA, Jones AE, Cox RD. Longitudinal trends in the prevalence of diabetes mellitus in an urban emergency department. South Med J 2016; 109: 222-7.

2. Minami Y, Kajimoto K, Sato N, Hagiwara N, Takano T. Endstage renal disease patients on chronic maintenance hemodialysis in a hospitalized acute heart failure cohort: Prevalence, clinical characteristics, therapeutic options, and mortality. Int J Cardiol 2016; 224: 267-70.

3. Genereux P, Madhavan MV, Mintz GS, et al. Relation between coronary calcium and major bleeding after percutaneous coronary intervention in acute coronary syndromes (from the Acute Catheterization and Urgent Intervention Triage Strategy and Harmonizing Outcomes With Revascularization and Stents in Acute Myocardial Infarction Trials). Am J Cardiol 2014; 113: 930-5.

4. Barbato E, Carrie D, Dardas P, et al. European expert consensus on rotational atherectomy. EuroIntervention 2015; 11: 30-6.

5. Tomey MI, Kini AS, Sharma SK. Current status of rotational atherectomy. Catheter Cardiovasc Interv 2014; 7: 345-53.

6. Yamamoto S, Sakakura K, Funayama H, Wada H, Fujita H, Momomura S. Percutaneous coronary artery bypass for type 3 coronary perforation. JACC Cardiovasc Interv 2015; 8: 1396-8.

7. Sakakura K, Ako J, Momomura S. Successful removal of an entrapped rotablation burr by extracting drive shaft sheath followed by balloon dilatation. Catheter Cardiovasc Interv 2011; 78: $567-70$.

8. Sakakura K, Funayama H, Taniguchi Y, et al. The incidence of slow flow after rotational atherectomy of calcified coronary arteries: A randomized study of low speed versus high speed. Catheter Cardiovasc Interv 2017; 89: 832-40.

9. Sakakura K, Taniguchi Y, Yamamoto K, Wada H, Momomura SI, Fujita $\mathrm{H}$. When a burr can not penetrate the calcified lesion, increasing burr size as well as decreasing burr size can be a solution in rotational atherectomy. Int Heart J 2017; 58: 279-82.

10. Sakakura K, Taniguchi Y, Matsumoto M, Wada H, Momomura SI, Fujita H. How should we perform rotational atherectomy to an angulated calcified lesion? Int Heart J 2016; 57: 376-9.

11. Sakakura K, Inohara T, Kohsaka S, et al. Incidence and determinants of complications in rotational atherectomy. Circ Cardiovasc Interv 2016; 9: e004278. 